\title{
Photoacoustic microscopy using Evans Blue dye as a contrast agent
}

Junjie Yao, Konstantin I. Maslov, Song Hu, Lihong V. Wang

Junjie Yao, Konstantin I. Maslov, Song Hu, Lihong V. Wang, "Photoacoustic microscopy using Evans Blue dye as a contrast agent," Proc. SPIE 7564, Photons Plus Ultrasound: Imaging and Sensing 2010, 75642J (23 February 2010); doi: 10.1117/12.840684

SPIE. Event: SPIE BiOS, 2010, San Francisco, California, United States 


\title{
Photoacoustic microscopy using Evans Blue dye
}

\author{
as a contrast agent \\ Junjie Yao, Konstantin I. Maslov, Song Hu, Lihong V. Wang* \\ Optical Imaging Laboratory, Department of Biomedical Engineering, \\ Washington University in St. Louis, One Brookings Dr., St. Louis, Missouri 63130, USA
}

\begin{abstract}
Complete and continuous imaging of microvascular networks is crucial for a wide variety of biomedical applications. Photoacoustic tomography can provide high resolution microvascular imaging using hemoglobin within red blood cells (RBC) as an endogenous contrast agent. However, intermittent RBC flow in capillaries results in discontinuous and fragmentary capillary images. To overcome this problem, we used Evans Blue (EB) dye as a contrast agent for in vivo photoacoustic imaging. EB has strong optical absorption at $610 \mathrm{~nm}$ and distributes uniformly in the blood stream by chemically binding to albumin. By intravenous injection of EB $(6 \%, 200 \mu \mathrm{L})$, complete and continuous microvascular networks - especially capillaries - of the ears of nude mice were imaged. The diffusion of EB $(3 \%, 100 \mu \mathrm{L})$ leaving the blood stream was monitored for 2 hours. At lower administration dose of $\mathrm{EB}(3 \%, 50 \mu \mathrm{L})$, the clearance of the EB-albumin complex was imaged for 10 days and quantitatively investigated using a two-compartment model.
\end{abstract}

Keywords: Optical-resolution photoacoustic microscopy, Evans Blue dye, Evans Blue-Albumin complex, diffusion dynamics

\section{INTRODUCTION}

Photoacoustic (PA) tomography, which combines the spatial resolution of ultrasound imaging with the contrast of optical absorption in deep biological tissues, ${ }^{1}$ has gained great attention in biomedical applications. ${ }^{2} \mathrm{PA}$ imaging is complementary with other high resolution optical imaging modalities such as confocal microscopy, two-photon microscopy, and optical coherence tomography, which can provide in vivo imaging within the optical transport mean free path $(\sim 1 \mathrm{~mm})$ of biological tissues. ${ }^{3}$

An optical-resolution photoacoustic microscope (OR-PAM) has been described previously, with a lateral resolution of $5 \mu \mathrm{m}$, axial resolution of $15 \mu \mathrm{m}$, and imaging depth greater than $0.7 \mathrm{~mm}$. ${ }^{4}$ OR-PAM is a good tool to image blood vessels on the capillary level using hemoglobin within red blood cells (RBC) as an endogenic contrast agent. However, RBC flow in capillaries is discontinuous and changes greatly over time. ${ }^{5}$ Because RBCs are the only noticeable optical absorbers in capillaries, it is highly likely that no absorber is present in a particular voxel during the laser pulse, which results in discontinuous capillaries in a RBC-based PA image. In this work we use Evans Blue (EB) dye for this purpose. EB has strong absorption in visible and near-infrared light, with a peak at $620 \mathrm{~nm}$. EB is non-toxic and is used in measurement of blood volume, ${ }^{6}$ lymph node location, ${ }^{7}$ microvascular permeability, ${ }^{8}$ among other applications. In the blood stream, EB mainly binds to serum albumin in a reversible manner, so it is uniformly distributed in the plasma, maximizing the chance to get a complete capillary network image. Under normal conditions, the EB-albumin (EBA) complex is confined within blood vessels, while the free dye more readily diffuses out into extravascular tissue. The diffused dye is bound to the surrounding tissue proteins, and finally cleared out by either metabolism or excretion. ${ }^{9}$

* Corresponding author: Lihong V. Wang, lhwang@biomed.wustl.edu

Photons Plus Ultrasound: Imaging and Sensing 2010, edited by Alexander A. Oraevsky, Lihong V. Wang, Proc. of SPIE Vol. 7564, 75642J · C 2010 SPIE · CCC code: 1605-7422/10/\$18 · doi: 10.1117/12.840684 
This work seeks to achieve complete capillary network imaging, to investigate the dynamic diffusion of EB out of the circulation system, and to quantitatively elucidate the dynamic clearance of EBA.

\section{MATERIALS AND METHODS}

\subsection{Animal preparation}

The ears of adult, 6- to 8-week-old nude mice (Hsd: Athymic Nude-Foxl ${ }^{\mathrm{NU}}$, Harlan Co.; body weight: $\sim 20$ g) were used for all in vivo experiments here. During data acquisition, the animal was placed on a heating pad $\left(37^{\circ} \mathrm{C}\right)$, and kept still by using a breathing anesthesia system (E-Z Anesthesia, Euthanex). After experiment, the animal recovered naturally and was returned to its cage. All experimental animal procedures were carried out in conformity with the laboratory animal protocol approved by the Animal Studies Committee of School of Medicine at Washington University in St. Louis.

\subsection{Mode of injection of EB}

A $6 \%$ or $3 \% \mathrm{~EB} w / \mathrm{v}$ solution (Sigma, St. Louis, $\mathrm{MO}$ ) was prepared by dilution of the dye in phosphatebuffered saline (PBS, pH 7.5). Before injection, the solution was filtered through a 5- $\mu \mathrm{m}$ filter. An intravenous injection of EB was made to either of the dorsal veins of the tail. The injection lasted for about $10-20$ seconds.

\section{SPATIALLY CONTINUOUS CAPILLARY IMAGING}

Two irradiation wavelengths $570 \mathrm{~nm}$ and $610 \mathrm{~nm}$ were chosen for RBC imaging and EB imaging, respectively. An area of $2 \mathrm{~mm} \times 2 \mathrm{~mm}$ was chosen as the field of interest near the margin of the nude mouse ear, where the capillary density was higher. Before the dye injection, control images were acquired with a scanning step size of $2.5 \mu \mathrm{m}$ at $570 \mathrm{~nm}$ [Fig. 1(b)] and $610 \mathrm{~nm}$ [Fig. 1(c)]. The total scanning time for a complete volumetric data set was $\sim 30$ min for each wavelength. In order to get sufficient imaging contrast and sensitivity of the capillaries, a relatively high concentration of EB in the blood plasma should be reached. Here $0.2 \mathrm{~mL}$ of $6 \%$ EB solution was injected in a nude mouse. Transmission optical microscopic images at $4 \times$ magnification were acquired before and after injection [Figs. 1(a) and (e)]. 

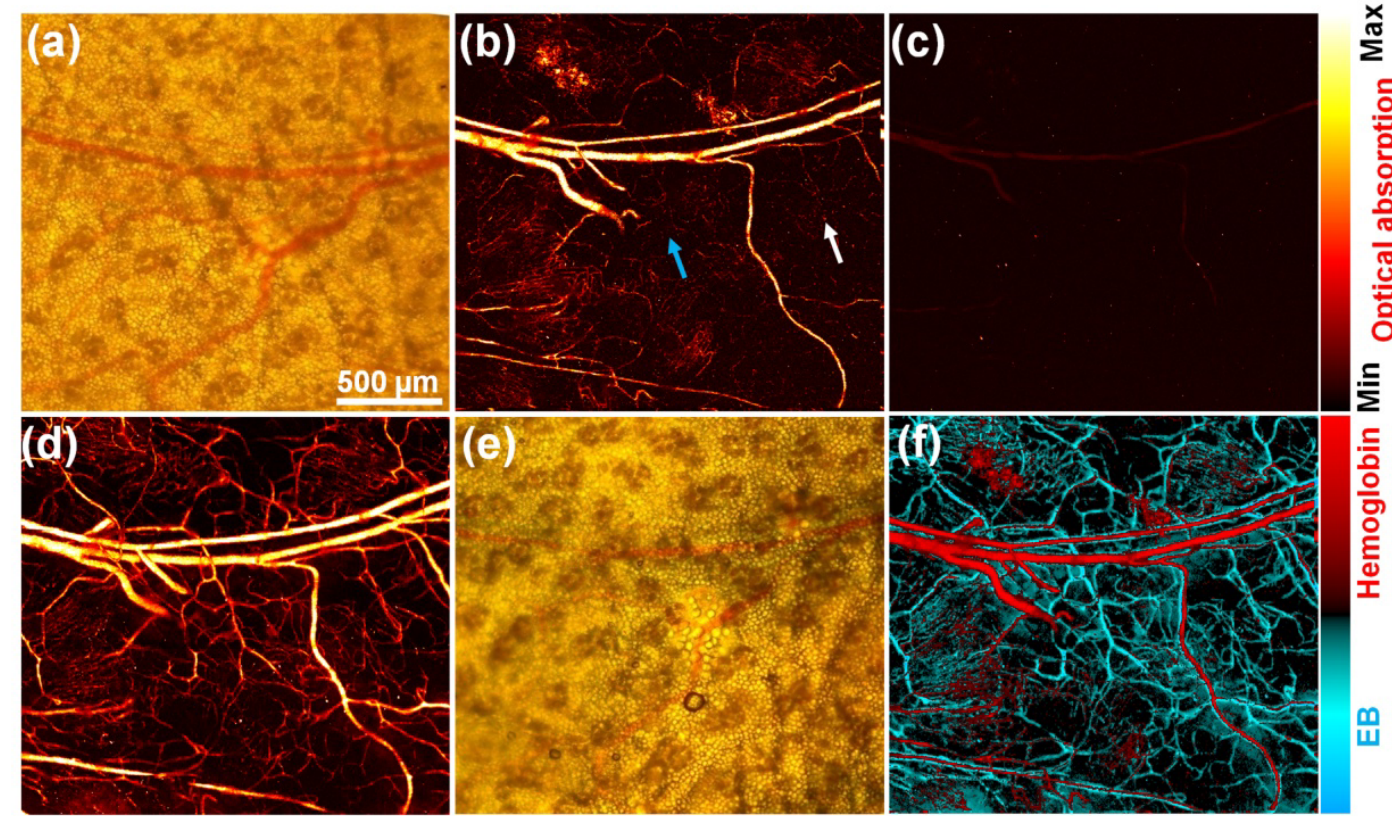

Figure 1. EB enhanced photoacoustic imaging of mouse ear microvessels. (a) Transmission microscopic image before dye injection. (b, c) PA microvascular image before dye injection acquired at $570 \mathrm{~nm}$ (a) and at $610 \mathrm{~nm}$ (b). Arrows in (b) point to the fragmentary capillaries. (d) PA image acquired at $610 \mathrm{~nm}$ right after $\mathrm{EB}(6 \%, 0.2 \mathrm{~mL})$ injection via tail vein. (e) Transmission microscopic image after dye injection. (f) Composite image of (b) and (d), where EB-based signal from (d) is shown in blue and the RBC-based signal from (b) is shown in red as a superposition. All the photoacoustic images are scaled to the same level of PA signal.

Right after the dye injection, the microvascular network appeared continuous as shown in Fig. 1(d). Dense capillaries could be observed. All the capillaries appear 'broken' in Fig. 1(b) became smooth and continuous. Moreover, the capillary branching points that were invisible in Fig. 1(b) could be clearly distinguished. The blood vessels in Fig. 1(d) appeared somewhat thicker than those in Fig. 1(b), which was possibly because the plasma volume was larger than the RBC volume. The discernable blood vessel volume in the plasma-based image appeared to be more than 50\% greater than that disclosed in the RBCbased image, as shown in Fig. 1(f), where EB-based signal is shown in blue and the original RBC-based signal is shown in red as a superposition.

\section{DYNAMICS OF EB DIFFUSION OUT OF THE BLOOD STREAM}

EB is removed from the vascular system principally by diffusing into extravascular tissue. At high dye concentrations, in the first few hours, it is mainly the free EB rather than the EB-albumin complex that diffuses out. To better understand the diffusion dynamics, we monitored the dye diffusion over time. Here, a smaller area of $1 \mathrm{~mm} \times 1 \mathrm{~mm}$ was imaged near the margin of the nude mouse ear [Fig. 2(a)], so more data sets could be acquired over time due to the shorter scanning time of $\sim 10 \mathrm{~min}$. Control images at $610 \mathrm{~nm}$ [Fig. 2(b)] was acquired before dye injection. After $0.1 \mathrm{~mL}$ of $6 \%$ EB solution was injected, serial images at $610 \mathrm{~nm}$ were acquired every $20 \mathrm{~min}$ until the dye diffusion was observed to have reached saturation. As composited in Figs. 2(c)-(h), the blood vessel signal from Fig. 2(c) is shown in red and EB signal outside the vessels is shown in blue. 

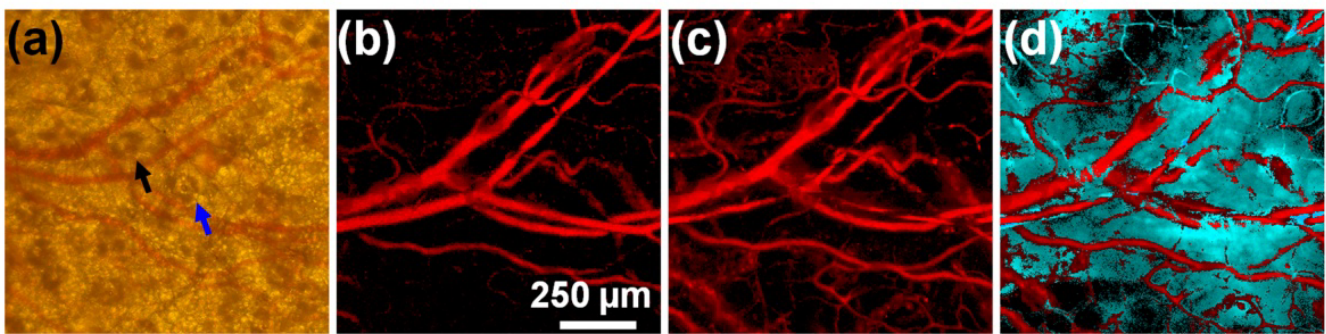

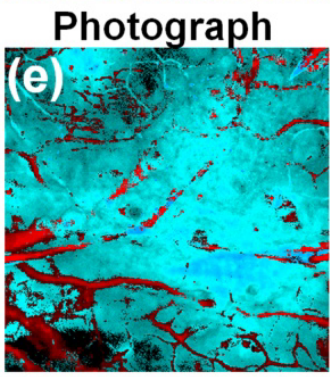

$50 \mathrm{~min}$

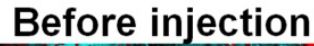

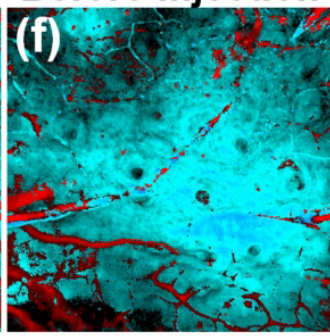

$70 \mathrm{~min}$
$10 \mathrm{~min}$

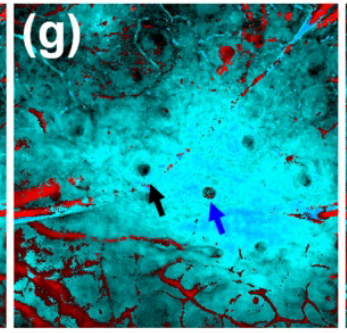

$90 \mathrm{~min}$

\section{$30 \mathrm{~min}$}

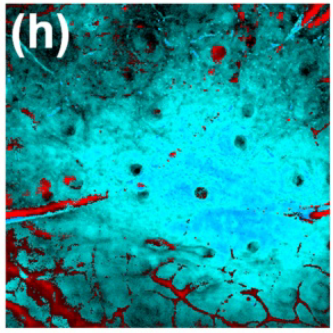

$120 \mathrm{~min}$

vessel EB

Figure 2. Dynamics of EB diffusion out of the blood stream into surrounding tissue. (a) Transmission microscopic image before dye injection. (b) PA image acquired before EB injection at $610 \mathrm{~nm}$. (c)-(h) Composite PA images acquired at $610 \mathrm{~nm}$ after $\mathrm{EB}(6 \%, 0.1 \mathrm{~mL})$ injection at different times. The blood vessel signal from (c) is shown in red and EB signal outside the vessels is shown in blue. Arrows in (a) and (g) point to sebaceous glands.

The partial volume of the free EB molecule diffused into extravascular tissue increased gradually and reached a plateau after $\sim 2$ hours. EB did not diffuse into the sebaceous glands, so more and more 'dark cavities' were formed as the diffusion went on, as shown by the arrows in Fig. 2(g). Blood vessels were embedded in the diffused dye and capillaries became nearly invisible.

\section{DYNAMICS OF THE EBA CLEARANCE}

At low dye concentrations, EB exists almost exclusively in the form of EBA. ${ }^{9}$ EB permeates wherever albumin is present. Therefore, the clearance dynamics of the EBA may be used to estimate the albumin metabolic rate in tissue. ${ }^{9}$ To better understand the clearance dynamics, the EBA volume in the tissue was monitored by PA imaging. An imaging area of $1 \mathrm{~mm} \times 1 \mathrm{~mm}$ [Fig. 3(a)] was chosen on the nude mouse ear, and a control image at $570 \mathrm{~nm}$ was acquired before dye injection [Fig. 3(b]. Then $0.05 \mathrm{~mL}$ of $3 \% \mathrm{~EB}$ solution was injected via the tail vein. Serial images [Figs. 3(c)-(j)] were acquired at $570 \mathrm{~nm}$ (shown in red) and $610 \mathrm{~nm}$ (shown in blue) every one or two days, until EBA had nearly completely cleared out. The EBA volume in the extravascular tissue reached maximum on Day 3 and then decreased to the base line by Day 10. A two-compartment model was used to fit the EBA volume in the extravascular tissue [Fig. 3(k)].

$$
\begin{aligned}
& \frac{d Q_{V}}{d t}=-k_{1} Q_{V} \\
& \frac{d Q_{T}}{d t}=k_{1} Q_{V}-k_{2} Q_{T}
\end{aligned}
$$

where $Q_{V}, Q_{T}$ are the EBA volumes in the blood stream and extravascular tissue, respectively. $k_{1}$ is the diffusion rate of EBA from the blood stream into the extravascular tissue, and $k_{2}$ is the clearance rate of EBA in the tissue. 

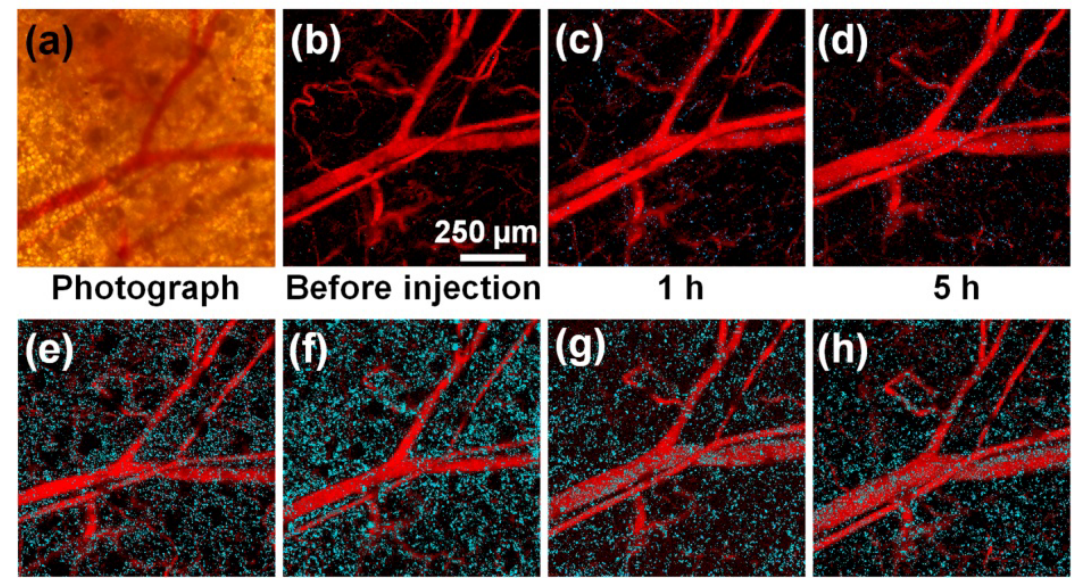

Day 1

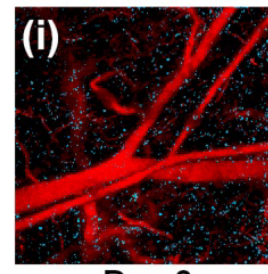

Day 8
Day 3

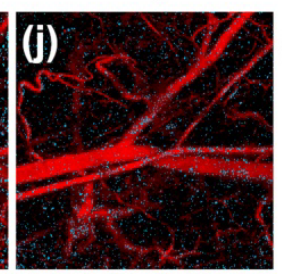

Day 10
Day 4

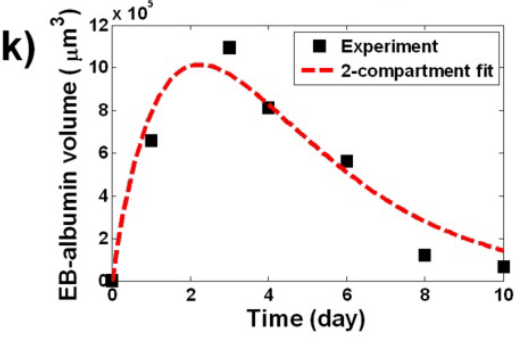

Vessel EB-albumin

Figure 3. Clearance dynamics of EBA. Before EB injection, transmission microscopic image (a) and PA image (b) at $570 \mathrm{~nm}$ were acquired. On $1 / 2-10$ days following EB $(3 \%, 0.05 \mathrm{~mL})$ injection, images (c)-(j) were acquired at $570 \mathrm{~nm}$ (shown in red) and $610 \mathrm{~nm}$ (shown in blue). All the photoacoustic images are scaled to the same level. (k) A twocompartment model was used to fit the EBA volume in the extravascular tissue.

\section{CONCLUSION}

In this work, we demonstrate the feasibility of using Evans Blue dye as a contrast agent to enhance in vivo photoacoustic microvascular imaging. Complete and continuous microvascular networks especially capillaries were imaged with the contrast of EB, and PA imaging has been used to study the dynamics of EB diffusion and EBA clearance.

\section{ACKNOWLEDGEMENT}

This research is supported by the National Institutes of Health Grants R01 EB000712, R01 NS46214, R01 EB008085 and U54 CA136398. Lihong V. Wang has a financial interest in Endra, Inc., which, however, did not support this work.

\section{REFERENCE}

[1]. M. H. Xu and L. H. V. Wang, "Photoacoustic imaging in biomedicine," Rev Sci Instrum. 77, pp. 041101, 2006.

[2]. L. V. Wang, "Prospects of photoacoustic tomography," Med Phys. 35, pp. 5758-5767, 2008.

[3]. L. V. Wang and H.-i. Wu, Biomedical optics : Principles and imaging, Wiley-Interscience, Hoboken, N.J, 2007.

[4]. K. Maslov, H. F. Zhang, S. Hu and L. V. Wang, "Optical-resolution photoacoustic microscopy for in vivo imaging of single capillaries," Opt Lett. 33, pp. 929-931, 2008.

[5]. E. Chaigneau, M. Oheim, E. Audinat and S. Charpak, "Two-photon imaging of capillary blood flow in olfactory bulb glomeruli," P Natl Acad Sci USA. 100, pp. 13081-13086, 2003. 
[6]. T. D. Poulsen, T. Klausen, J. P. Richalet, I. L. Kanstrup, N. Fogh-Andersen and N. V. Olsen, "Plasma volume in acute hypoxia: Comparison of a carbon monoxide rebreathing method and dye dilution with evans' blue," Eur J Appl Physiol O. 77, pp. 457-461, 1998.

[7]. M. I. Harrell, B. M. Iritani and A. Ruddell, "Lymph node mapping in the mouse," J Immunol Methods. 332, pp. 170-174, 2008.

[8]. W. G. Roberts and G. E. Palade, "Increased microvascular permeability and endothelial fenestration induced by vascular endothelial growth-factor," J Cell Sci. 108, pp. 2369-2379, 1995.

[9]. H. H. LeVeen and W. H. Fishman, "Combination of evans blue with plasma protein: Its significance in capillary permeability studies, blood dye disappearance curves, and its use as a protein tag," Am J Physiol. 151, pp. 26-33, 1947. 